\title{
PROPIEDADES DE TABLEROS AGLOMERADOS DE PARTICULAS FABRICADOS CON MADERA DE Eucalyptus urophylla
}

\author{
PROPERTIES PARTICLEBOARDS MANUFACTURED WITH \\ Eucalyptus urophylla WOOD
}

\author{
Leidig Rangel ${ }^{1}$, Pablo Moreno ${ }^{2,4}$, Shakespeare Trejo ${ }^{2}$, Styles Valero ${ }^{2}$
}

\begin{abstract}
RESUMEN
Este es un estudio exploratorio para dar uso a la madera de Eucalyptus urophylla en la elaboración de tableros aglomerados de partículas. Por lo tanto, los objetivos fueron determinar sus propiedades físico-mecánicas y evaluar los resultados bajo norma Alemana Instituto Alemán de Normalización (DIN) y Venezolana Comisión Venezolana de Normas Industriales (CONEVIN). Para los tableros de densidad real de $731 \mathrm{~kg} / \mathrm{m}^{3}$, la absorción de agua promedio a 2 y 24 horas no cumple de acuerdo a las normas, para el caso de los tableros de densidad real $831 \mathrm{~kg} / \mathrm{m}^{3}$ cumple con la norma. La variación del espesor (hinchamiento) promedio por inmersión en agua a 2 horas para los tableros con densidad de $731 \mathrm{~kg} / \mathrm{m}^{3}$ no cumple con lo exigido en ambas normas, pero si cumple con los requerimientos para 24 horas. Para el caso de los tableros con densidad de $838 \mathrm{~kg} / \mathrm{m}^{3}$ la variación de espesor a 2 y 24 horas cumplió con las normas. Respecto de las propiedades mecánicas, tanto el módulo de ruptura en flexión estática como la tensión perpendicular (adhesión interna) para ambos niveles de densidad superaron el valor mínimo aceptado en las normas, demostrando que las partículas de madera de Eucalyptus urophylla presentan características tecnológicas adecuadas para la fabricación de tableros de partículas de densidad media.
\end{abstract}

Palabras clave: Adhesión interna, densidad, flexión estática, tensión perpendicular, urea formaldehído.

\begin{abstract}
This is an exploratory study on the feasibility of using Eucalyptus urophylla wood for the production of particleboards. Therefore, the aims were to determine their physical and mechanical properties and to evaluate the results with German Standards Institute (DIN) and Industrial Standards Commission Venezuelan (COVENIN). For boards with a real density of $731 \mathrm{~kg} / \mathrm{m}^{3}$, the average water absorption at 2 and 24 hours does not meet the standards, in the case of real density boards $831 \mathrm{~kg} / \mathrm{m}^{3}$ that fulfilled with both standards. The average water absorption at 2 and 24 hours of boards for the actual density of 731 $\mathrm{kg} / \mathrm{m}^{3}$ did not comply with the requirements for both standards, unlike the particleboards with density equal $831 \mathrm{~kg} / \mathrm{m}^{3}$ that fulfilled with them. The average thickness variation (swelling) by immersion in water for 2 hours for boards with a density of $731 \mathrm{~kg} / \mathrm{m}^{3}$ did not meet the requirements of both
\end{abstract}

\footnotetext{
1'Instituto Universitario Santiago Mariño. Mérida-Venezuela. leidigrangel@hotmail.com

${ }^{2}$ Universidad de Los Andes, Facultad de Ciencias Forestales y Ambientales. Laboratorio Nacional de Productos Forestales.

Mérida, Venezuela.shakespeare@ula.ve, styles@ula.ve

•Autor para correspondencia: pmoreno@ula.ve

Recibido: 26.09.2016 Aceptado: 27.04.2017
} 
standards, but meet the requirements for 24 hours. In the case of boards with a density of $838 \mathrm{~kg} / \mathrm{m}^{3}$, thickness variation at 2 and 24 hours meet with the standards. Regarding mechanical properties both, the modulus of rupture in static bending and perpendicular tension (internal bond) for both real density levels exceed the minimum accepted by standards, demonstrating that E. urophylla wood exhibit suitable technological characteristics for particleboards medium density manufacture.

Keywords: Density, internal bond, perpendicular tension, static bending, urea formaldehyde.

\section{INTRODUCCIÓN}

En Venezuela, la industria de contrachapado y aglomerado se inicia en el año 1957, con la puesta en marcha de la fábrica Cariban, también denominada Madepren. Para el año 1987, de las 10 plantas existentes en el país sólo 8 estaban en producción con una capacidad instalada de $250000 \mathrm{~m}^{3} / \mathrm{año} \mathrm{y}$ la producción global representaba solo el $40 \%$ de la capacidad instalada; para la fecha 2016, se cuenta con solo dos empresas que fabrican tableros aglomerados de partículas y de fibra.

La industria de los tableros aglomerados de partículas en Venezuela utilizaba como materia prima principalmente ramas y madera rolliza de pequeñas dimensiones provenientes del aprovechamiento primario en el bosque y residuos de industrias forestales, como aserraderos, carpinterías, fábricas de contrachapados, entre otras.

La utilización excesiva de especies latifoliadas y de alto valor comercial ha generado que estas especies estén en programa de recuperación o veda, afectando esta medida el sistema productivo del sector de tableros aglomerados en el país, y en muchos de los casos ha llevado al cierre definitivo o temporal de estas empresas por falta de materia prima.

En vista de esto, algunas empresas para mantenerse en el mercado nacional han optado por utilizar otras especies como la madera de plantaciones de coníferas (pino caribe) de rápido crecimiento, materia prima que se encuentra muy retiradas del sitio de producción de los tableros, lo cual aumenta los costos por traslado o flete de estas especies desde las plantaciones hasta la fábrica.

Mendes et al. (2014) indican que las perspectivas son bastantes positivas para el crecimiento a nivel mundial del sector de tableros de madera de forma general, siendo los tableros de partículas el de mayor consumo (57\%) del total, con un crecimiento continuo entre un $2 \%$ a $5 \%$ anualmente (Ashoria y Nourbakhsh 2008, Kord et al. 2016).

Venezuela presenta un potencial de plantaciones de Pinus caribaea (pino caribe) de aproximadamente 650000 ha, en menor proporción el género Eucalyptus con aproximadamente 17855 ha, de las cuales 12135 ha son manejadas por el sector público a través de Maderas del Orinoco, plantadas en los estados Anzoátegui, Bolívar y Monagas (Gonzales 2016), y de 5720 ha plantadas en la finca Desarrollo Forestal San Carlos II (Deforsa), manejadas de forma privada, para ser utilizadas con fines industriales para la obtención de pulpa para papel; y madera para elaborar paletas, estantillos, entre otros productos y subproductos.

Con el crecimiento de la industria de tableros de madera, aumenta también la demanda de materia prima, lo que es necesario un aumento de las áreas de plantación con pino caribe, especie que en los últimos 20 años viene siendo utilizada como materia prima para la fabricación de tableros aglomerados de partículas y fibras, además de la búsqueda de otras especies de rápido crecimiento que puedan contribuir de forma cuantitativa y cualitativa para suplir las necesidades, y de esta manera poder reactivar y establecer nuevas empresas del sector de tableros a base de madera en Venezuela.

De acuerdo con Trianosky (2010), algunas especies no convencionales de rápido crecimiento, tienen elevado potencial para producción de tableros de madera aglomerada, las mismas provienen de plantaciones experimentales o de pequeñas aéreas forestales, muy incipientes para suplir las necesidades industriales.

Una de las alternativas potenciales para solucionar la problemática existente por la falta de materia prima y poder reactivar el sector de los tableros aglomerados en el país, es incorporar 
especies consideradas de rápido crecimiento, presentando ventajas en términos de mayor incremento volumétrico. En base al número de hectáreas plantadas, el Eucaliptus urophylla se destaca y podría convertirse en una alternativa como materia prima para tableros aglomerados de partículas y que podría abastecer cualquier demanda; de esta manera, se ampliarían sus usos incorporándose satisfactoriamente en el mercado nacional.

Basado en los resultados positivos de estas investigaciones en otros países y con el propósito de determinar la factibilidad tecnológica de la especie Eucalyptus urophylla en Venezuela para la fabricación de tableros de partículas, se desarrolló en el presente estudio una investigación de tipo exploratorio con el fin de determinar sus propiedades físicas y mecánicas.

\section{MATERIALES Y MÉTODOS}

Como materia prima, se utilizó madera de la especie Eucalyptus urophylla de 10 años de edad, proveniente de las plantaciones de la Empresa Agroforestal Anzoátegui C.A, estado AnzoáteguiVenezuela. La elaboración de los tableros, la preparación de probetas y la ejecución de los ensayos se realizaron en el Laboratorio Nacional de Productos Forestales: Sección tableros aglomerados y ensayos de la Facultad de Ciencias Forestales y Ambientales de la Universidad de Los Andes, MéridaVenezuela.

\section{Determinación de la densidad y contenido de humedad de la materia prima}

Se seleccionaron trozas con un diámetro aproximado de $9 \mathrm{~cm}$ y se cortaron segmentos de $18 \mathrm{~cm}$ de longitud, de cada troza y de forma aleatoria se eligieron segmentos, se prepararon 30 probetas con dimensiones de $30 \mathrm{~mm}$ x $30 \mathrm{~mm}$ x $100 \mathrm{~mm}$ para la determinación de la densidad en las condiciones verde, seca al aire y seca al horno, según la norma DIN (1976). El cálculo del contenido de humedad se realizó con la siguiente ecuación:

$$
\mathrm{CH}=\left(\frac{\mathrm{Pi}-\mathrm{Po}}{\mathrm{Po}}\right) \times 100
$$

Dónde:

$\mathrm{CH}$ : contenido de humedad $(\%)$

$\mathrm{P}_{\mathrm{i}}$ : Masa inicial de la muestra $(\mathrm{g})$

$\mathrm{P}_{\mathrm{o}}$ : Masa anhidra de la muestra $(\mathrm{g})$

\section{Viruteado de la madera de $E$. urophylla y secado de partículas}

Los segmentos de madera fueron procesados en una viruteadora de cuchillos para obtener partículas con una dimensión promedio de $20 \mathrm{~mm}$ de longitud, ancho variable y espesor de $0,25 \mathrm{~mm}$. Desde la viruteadora las partículas fueron transferidas por un sistema neumático de ciclones a la secadora de suspensión Keller, donde fueron secadas a un contenido de humedad final de $4 \%$.

\section{Coeficiente de esbeltez}

De manera aleatoria se tomaron $30 \mathrm{~g}$ de las partículas secas para determinar su geometría y tamaño, éstas se clasificaron de manera visual en tres categorías de tamaño: grandes, medianas y pequeñas; cada categoría fue pesada y se tomaron 30 muestras de cada una. Con un vernier digital de $0,02 \mathrm{~mm}(0,0001$ 
pulgadas) de resolución, se midió largo, ancho y espesor de las partículas y se determinó su coeficiente de esbeltez (E), con la ecuación (2). La proporción de participación de cada categoría de tamaño de partícula para conformar el tablero, se determinó mediante su peso.

$$
E=\left(\frac{L}{e}\right)
$$

Donde:

E: coeficiente de esbeltez

L: longitud de las partículas (mm)

E: espesor de las partículas (mm)

\section{Componentes para la fabricación de los tableros}

Se utilizaron los siguientes insumos: Partículas de E. urophylla a $4 \%$ de contenido de humedad, adhesivo urea formaldehído (UF) a $60 \%$ de sólidos con viscosidad $870 \mathrm{cP}, \mathrm{pH} 8,5$ y como catalizador una solución de sulfato de amonio al $30 \%(\mathrm{p} / \mathrm{p})$ de concentración, la cantidad de catalizador corresponde al $7 \%(\mathrm{p} / \mathrm{p})$ de la cantidad de adhesivo aplicada a cada tablero. Se fabricaron 5 tableros homogéneos para dos niveles de densidad teórica de $750 \mathrm{~kg} / \mathrm{m}^{3}$ y $850 \mathrm{~kg} / \mathrm{m}^{3}$.

\section{Encolado de las partículas}

El encolado de las partículas secas a $4 \%$ de humedad se realizó por el método de aspersión a 0,203 $\mathrm{MPa}(2 \mathrm{~atm})$, por un tiempo de 8 minutos en una encoladora Drais, usando una carga de adhesivo UF al $12 \%$ de resinosidad respecto al peso seco de las partículas.

\section{Formación del colchón, proceso de pre-prensado y prensado del tablero de partículas}

Luego de encoladas las partículas fueron distribuidas manualmente sobre una lámina de aluminio, previamente parafinada y humectada con agua. Para retener las partículas en todos los costados del colchón se utilizó un marco o formaleta de madera. Una vez conformado el colchón de partículas se aplicó una carga de aproximadamente $785 \mathrm{~N}(80 \mathrm{kgf})$ a temperatura ambiente con la finalidad de compactar y disminuir el espesor, evitando la deformación del colchón de partículas en el proceso de prensado. Finalmente, para obtener los dos niveles de densidad en los tableros de partículas se aplicaron los respectivos ciclos de prensado (Figura 1) en una prensa Becker van Huller bajo las siguientes condiciones operacionales: Presión específica sobre el tablero: $3,05 \mathrm{MPa}\left(31,14 \mathrm{~kg} / \mathrm{cm}^{2}\right)$, presión manométrica de la prensa simple: $14,71 \mathrm{MPa}\left(150 \mathrm{~kg} / \mathrm{cm}^{2}\right)$, temperatura de los platos de la prensa: $180^{\circ} \mathrm{C}$ a $200^{\circ} \mathrm{C}$, tiempo de prensado total: 9 min para densidad teórica $750 \mathrm{~kg} / \mathrm{m}^{3}$ y $18 \mathrm{~min}$ para densidad teórica $850 \mathrm{~kg} / \mathrm{m}^{3}$. 


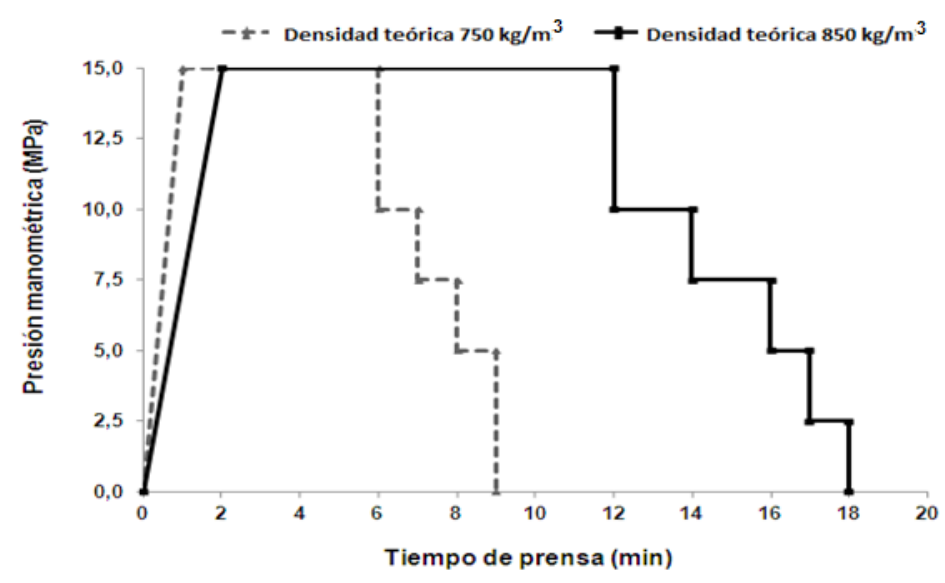

Figura 1. Diagrama del ciclo de prensado aplicado para densificar los tableros homogéneos de partículas de Eucalyptus urophylla a las densidades teóricas propuestas.

\section{Acondicionamiento y dimensionado de los tableros}

Los tableros fabricados fueron almacenados en un cuarto de acondicionamiento a $20^{\circ} \mathrm{C} \pm 1{ }^{\circ} \mathrm{C}$ y una humedad relativa de $65 \% \pm 2 \%$. Los tableros permanecieron bajo esas condiciones hasta que alcanzaron un contenido de humedad de equilibrio de $12 \%$ aproximadamente. Luego los tableros fueron dimensionados en una sierra circular con escuadría de $500 \mathrm{~mm}$ x $500 \mathrm{~mm}$, conservando el espesor de prensado de $19 \mathrm{~mm}$.

\section{Ensayos físicos y mecánicos de los tableros}

A partir de los tableros dimensionados se obtuvieron 10 probetas para cada uno de los ensayos, según las normas DIN (1965a, 1965b, 1965c, 1965d, 1965e). Los ensayos físicos fueron: contenido de humedad, densidad del tablero, absorción por inmersión en agua y variación de espesor por inmersión en agua a 2 y 24 horas. Los ensayos mecánicos fueron: resistencia a flexión estática (módulo de ruptura MOR) y tensión perpendicular a la superficie del tablero (adhesión interna) usando una máquina universal Instron y una velocidad de ensayo de $5 \mathrm{~mm} / \mathrm{min}$ para ambos ensayos. La evaluación de las propiedades físico-mecánicas de los tableros se realizó según lo estipulado por la norma venezolana COVENIN (1991) y alemana DIN (1981), utilizadas para tableros de partículas de madera de uso general con prensado plano.

\section{Diseño experimental}

Se utilizó un diseño con un solo factor (densidad) con 2 niveles categóricos y seis variables respuesta (absorción de agua a $2 \mathrm{~h}$ y $24 \mathrm{~h}$, variación de espesor o hinchamiento a $2 \mathrm{~h}$ y 24 h, módulo de ruptura en flexión estática y tensión paralela a la superficie del tablero). El análisis de varianza (ANOVA) se realizó con un nivel de confianza $\propto=0,05$ y con el software estadístico Design Expert $^{\circledR}$ V 8.0. 


\section{RESULTADOS Y DISCUSIÓN}

\section{Análisis de varianza (ANOVA) de un solo factor}

El análisis de varianza (Tabla 1) indica que el factor densidad del tablero es significativo para todas las variables respuesta. La probabilidad normal de residuales de los datos obtenidos en todas las variables respuesta cumple con el supuesto de normalidad, no se observaron fluctuaciones significativas de las muestras ensayadas ni datos atípicos. La proporción de variabilidad de cada parámetro respuesta es explicada por el modelo estadístico mediante el valor de $\mathrm{R}^{2}$. Por otra parte, el valor $\mathrm{R}^{2}$ ajustado indica que el número de factores presentes en el modelo es adecuado, por lo tanto, ambos confirman la validez del ANOVA.

Tabla 1. Valor- $p$ para las variables respuesta.

\begin{tabular}{|c|c|c|c|c|c|c|}
\hline \multirow{2}{*}{ Factor } & \multicolumn{7}{|c|}{${\text { Valor- } \boldsymbol{p}^{*}(\boldsymbol{\alpha}=\mathbf{0 , 0 5})}$} & ABS 2h & ABS 24h & VE 2h & VE 24h & MOR & TPS \\
\hline Modelo & $<0,0001$ & $<0,0001$ & 0,0001 & 0,0001 & $<0,0001$ & 0,0004 \\
\hline Densidad & $<0,0001$ & $<0,0001$ & 0,0001 & 0,0001 & $<0,0001$ & 0,0004 \\
\hline $\mathrm{R}^{2}$ & 0,9806 & 0,9552 & 0,2169 & 0,7305 & 0,1774 & 0,1210 \\
\hline $\mathrm{R}^{2}$ ajustado & 0,9804 & 0,9547 & 0,2089 & 0,7277 & 0,1690 & 0,1120 \\
\hline
\end{tabular}

$\mathrm{ABS}=$ absorción de agua a 2 y $24 \mathrm{~h} ; \mathrm{VE}=$ variación de espesor a 2 y 24 h; MOR= módulo de ruptura en flexión estática; TPS= tensión perpendicular a la superficie del tablero

* Valor- $p<0,05$ indica que los términos del modelo son significativos.

* Valor- $p>0,05$ indica que los términos del modelo no son significativos.

\section{Densidad de la madera de $E$. urophylla y tableros de partículas}

Los valores de la densidad promedio obtenidos para madera sólida utilizada como materia prima para la fabricación de tableros aglomerados de partículas, se presentan en la tabla 2. Los valores reales de densidad, así como sus coeficientes de variación para los tableros se muestran en la tabla 3 . Una comparación entre la densidad teórica y la real de los tableros de partículas y madera sólida de $E$. urophylla se presenta en la figura 2 .

Tabla 2. Densidad promedio para la madera sólida de Eucalyptus urophylla utilizada para la fabricación de tableros aglomerados de partículas.

\begin{tabular}{|l|c|c|c|}
\hline \multicolumn{1}{|c|}{ Condición } & $\begin{array}{c}\text { Densidad promedio } \\
\left(\mathbf{k g} / \mathbf{m}^{\mathbf{3}}\right)\end{array}$ & $\begin{array}{c}\text { Coeficiente de } \\
\text { variación } \mathbf{( \% )}\end{array}$ & $\begin{array}{c}\text { Contenido de } \\
\text { humedad (\%) }\end{array}$ \\
\hline Verde & 964 & 8,8 & 75,12 \\
\hline Seca al aire & 670 & 13,50 & 18,50 \\
\hline Seca al horno & 635 & 14,16 & - \\
\hline
\end{tabular}


Tabla 3. Densidad real promedio y coeficiente de variación de los tableros aglomerados de partículas de Eucalyptus urophylla.

\begin{tabular}{|c|c|}
\hline $\begin{array}{c}\text { Densidad real promedio } \\
\left(\mathbf{k g} / \mathbf{m}^{\mathbf{3}}\right)\end{array}$ & $\begin{array}{c}\text { Coeficiente de Variación } \\
\mathbf{( \% )}\end{array}$ \\
\hline 731 & 4,20 \\
\hline 838 & 1,52 \\
\hline
\end{tabular}

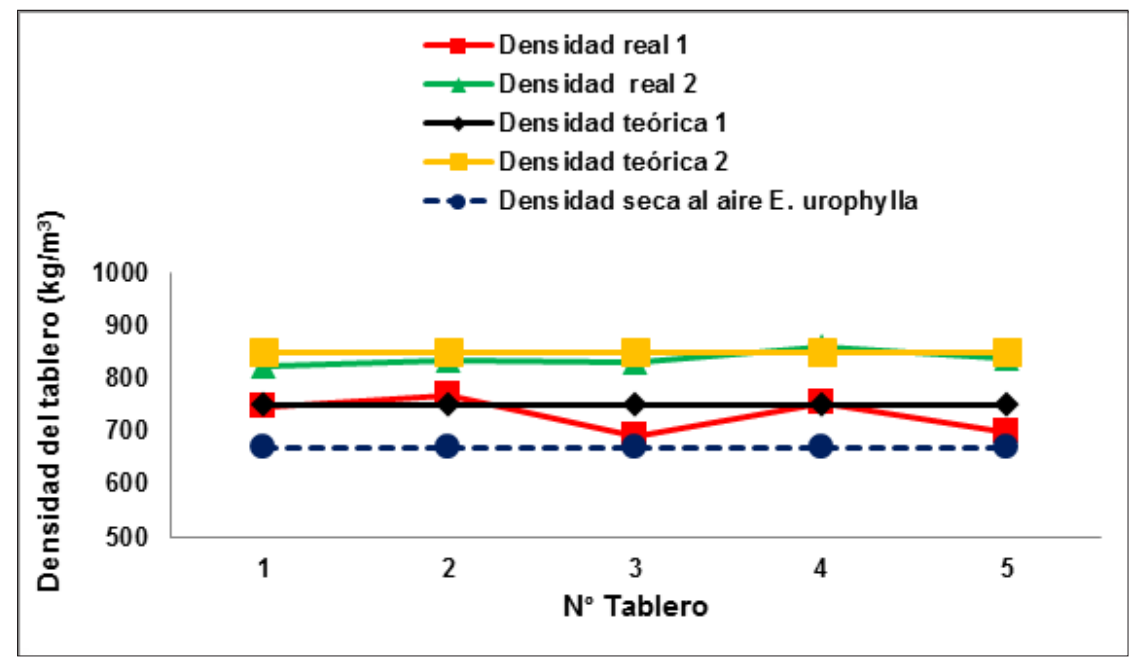

Figura 2. Comparación entre densidad teórica y real obtenida para los tableros homogéneos de partículas y la densidad de la madera sólida seca al aire de Eucalyptus urophylla.

El ciclo de prensado permitió obtener tableros con valores superiores a la densidad de la madera seca al aire de E. urophylla. Las densidades reales obtenidas de los tableros para ambas densidades, se encuentran por encima del valor de la densidad seca al aire de la madera de E. urophylla, situación que se considera conveniente cuando se fabrican tableros aglomerados de partículas, ya que al producir el tablero se comprimen las partículas a una densidad mayor que la que presentaban en el estado natural, siendo esta situación respaldada por Poblete et al. (2000), quienes mencionan que se debe tener presente que, para obtener tableros con propiedades adecuadas, la densidad del tablero debe ser mayor que la de la madera, presentando una razón de compresión mayor a 1,0. Sin embargo, las densidades reales o aparentes de los tableros se situaron por debajo de la densidad nominal estipulada de $750 \mathrm{~kg} /$ $\mathrm{m}^{3}$ y $850 \mathrm{~kg} / \mathrm{m}^{3}$. Esto puede ser justificado debido a la pérdida de partículas durante la formación del colchón, así como también puede estar relacionado con el retorno en espesor de los tableros al retirarlo de la prensa y colocarlos en acondicionamiento, con un consecuente aumento del volumen y reducción de la densidad nominal inicial del tablero (Mendes et al. 2014).

Cabral et al. (2007) fabricaron tableros aglomerados de partículas con tres especies Eucalyptus grandis, Eucalyptus urophylla e Eucalyptus cloeziana, y obtuvieron una media en densidad de 700 $\mathrm{kg} / \mathrm{m}^{3}$, valores que son similares a los obtenidos en la presente investigación para los tableros de densidad real $731 \mathrm{~kg} / \mathrm{m}^{3}$.Sin embargo, aun cuando en la conformación del producto participe una sola especie, la variabilidad de la densidad dentro y entre tableros es posible, situación que mencionan en su investigación Moreno et al. (2005) con tableros de partículas de ramas de Fraxinus americana y Zambrano et al. (2013) con residuos industriales de la madera de Pino patula. No obstante, los coeficientes de variación determinados para la densidad de los tableros de E. urophylla indican una buena distribución de las partículas en el producto final, de acuerdo con lo propuesto por Durán 1981, Garay 1988, Moreno 2002, Zambrano et al. 2013. 
La densidad del tablero, es una medida de la compactación individual de una partícula dentro del tablero y es dependiente, principalmente, de la densidad de la madera y la presión aplicada durante el prensado (Rocha et al. 1974). Un incremento en la densidad del tablero resulta en una mejor eficiencia de la resina, debido a la mejora de uniones adhesivas entre partícula y adhesivo (Gatchell et al. 1966). Poblete y Peredo (1990) lograron establecer, para tableros de partículas con especies nativas chilenas, relaciones lineales entre la propiedad de flexión y densidad, logrando producir tableros de partículas con densidad de $650 \mathrm{~kg} / \mathrm{m}^{3}$ que cumplieron con el valor mínimo de $15,99 \mathrm{MPa}\left(163 \mathrm{~kg} / \mathrm{cm}^{2}\right)$ solicitado por la norma DIN (1965c).

\section{Coeficiente de esbeltez}

Los valores promedio de participación de cada intervalo de partículas de E. urophylla seleccionado y su respectivo coeficiente de esbeltez se muestran en la tabla 4. El valor promedio del coeficiente de esbeltez para las partículas de E. urophylla $(122,73)$ se encuentra dentro del intervalo de esbeltez comprendido entre 120 y 200, intervalo que es considerado por Moslemi (1974), Medina (1975), Peredo (1988), Garay (1997), Moreno et al. (2002), Zambrano et al. (2013) como óptimo para la elaboración de tableros de partículas con propiedades físicas y mecánicas aceptables.

Tabla 4. Coeficiente de esbeltez (E) de las partículas de Eucalyptus urophylla.

\begin{tabular}{|c|c|c|c|c|}
\hline $\begin{array}{c}\text { Rango de tamaño en ancho } \\
(\mathbf{m m})\end{array}$ & $\begin{array}{c}\text { Proporción en } \\
\text { peso }(\mathbf{\%})\end{array}$ & $\begin{array}{c}\text { Largo } \\
(\mathbf{m m})\end{array}$ & $\begin{array}{c}\text { Espesor } \\
\mathbf{( m m})\end{array}$ & $\mathbf{E}$ \\
\hline$>10$ & 60 & 19,7 & 0,19 & 106,27 \\
\hline $5-10$ & 30 & 19,1 & 0,19 & 108,70 \\
\hline$<5$ & 10 & 19,0 & 0,14 & 153,23 \\
\hline Promedio & $\mathbf{1 0 0}$ & $\mathbf{1 9 , 3}$ & $\mathbf{0 , 1 7}$ & $\mathbf{1 2 2 , 7 3}$ \\
\hline
\end{tabular}

\section{Absorción de agua por inmersión}

En las figuras 3 a y 3 b se presenta la absorción de agua por inmersión a 2 y 24 horas de los tableros a ambas densidades. Los tableros con densidad de $838 \mathrm{~kg} / \mathrm{m}^{3}$ se encuentran de acuerdo a las especificaciones de la norma, sin embargo, los tableros con densidad de $731 \mathrm{~kg} / \mathrm{m}^{3}$ no cumplen con lo solicitado por la norma COVENIN (1991), que solicita $25 \%$ y $60 \%$ a 2 horas y 24 horas, respectivamente.
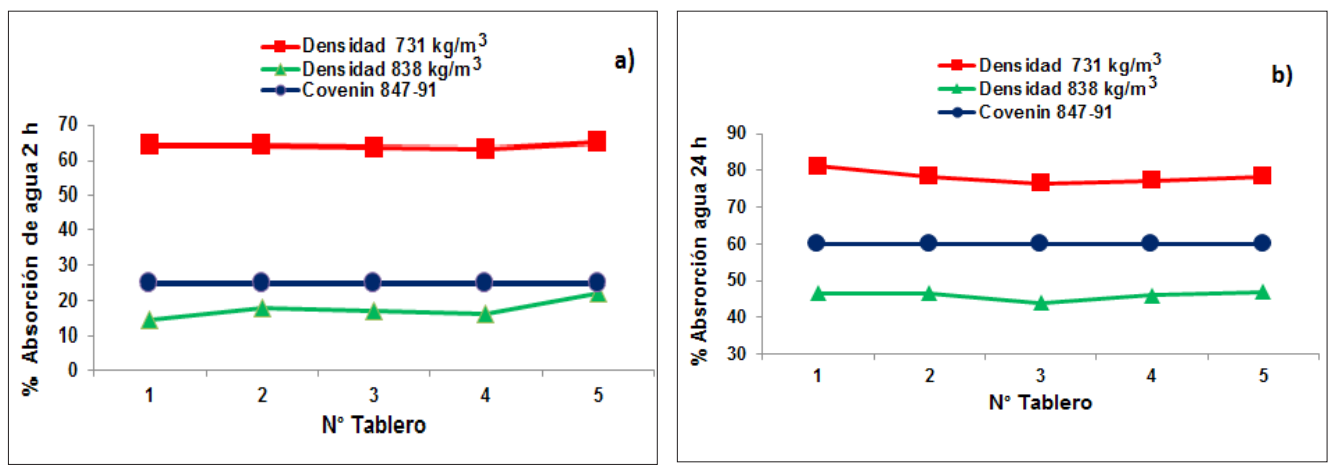

Figura 3. Absorción de agua por inmersión a) 2 h b) 24h, para tableros homogéneos de partículas de Eucalyptus urophylla. De acuerdo a las normas COVENIN (1991) y DIN (1981). 
Independiente del tiempo de inmersión, el incremento de la densidad del tablero produce una disminución en la absorción de agua, comportamiento que señalan Rocha et al. (1974), Durán (1982), Poblete y Peredo (1990), Moreno et al. (2002), Zambrano et al. (2013) para tableros de partículas. $\mathrm{Al}$ obtener un mayor contacto entre las partículas, se produce una disminución en la porosidad del tablero y menor cantidad de espacio vacío entre partículas y adhesivo es formado, lo que impide la penetración del agua. Así lo demuestran las investigaciones de Durán (1981 y 1982), Poblete y Peredo (1990), Maloney (1993), Moreno et al. (2005), quienes indican que la facilidad de absorción de agua está en función de la relación de compresibilidad del tablero, eficiencia del adhesivo y la facilidad de penetración del fluido (permeabilidad del tablero) por los espacios existentes entre partículas. Relaciones de compresibilidad bajas permiten al tablero absorber más humedad en comparación con tableros con alta tasa de compresibilidad.

Los tableros encolados con adhesivo UF no son aptos para uso a la intemperie (Peredo y Torres 1991) una exposición prolongada a la humedad lleva a la ruptura de los enlaces que forman la adhesión entre los grupos activos de la madera (partículas) y el adhesivo (Stark et al. 2010) afectando sus propiedades físico-mecánicas. Un calor excesivo también produce una degradación química del fraguado de la resina UF, por lo que, típicamente los tableros son enfriados rápidamente luego de finalizado el ciclo de prensado (Stark et al. 2010).

Los porcentajes de absorción de agua obtenidos para 2 y 24 horas de inmersión están en concordancia con los que exponen Bianche et al. (2012), para tableros de partículas de E. urophylla, Schizolobium amazonicum y Sida spp, con densidades reales de tableros de $630 \mathrm{~kg} / \mathrm{m}^{3}$, presentando un 17,41 $\%$ para 2 horas y $57,62 \%$ para 24 horas de inmersión en agua.

\section{Variación de espesor por inmersión en agua}

En las figuras 4a y $4 \mathrm{~b}$ se presenta la variación de espesor por inmersión en agua a 2 y 24 horas de los tableros con ambas densidades y de acuerdo con las normas COVENIN (1991) y DIN (1981) que solicitan una variación no mayor a $6 \%$ y $15 \%$ a 2 horas y 24 horas, respectivamente. Los tableros con densidad de $731 \mathrm{~kg} / \mathrm{m}^{3}$ no cumple con la exigencia de la norma COVENIN (1991) para la variación de espesor por inmersión en agua a 2 horas, pero cumple los requerimientos para la variación de espesor a 24 horas. Los tableros con densidad de $838 \mathrm{~kg} / \mathrm{m}^{3}$ cumple con lo solicitado por las normas para la variación de espesor por inmersión en agua a 2 y 24 horas.
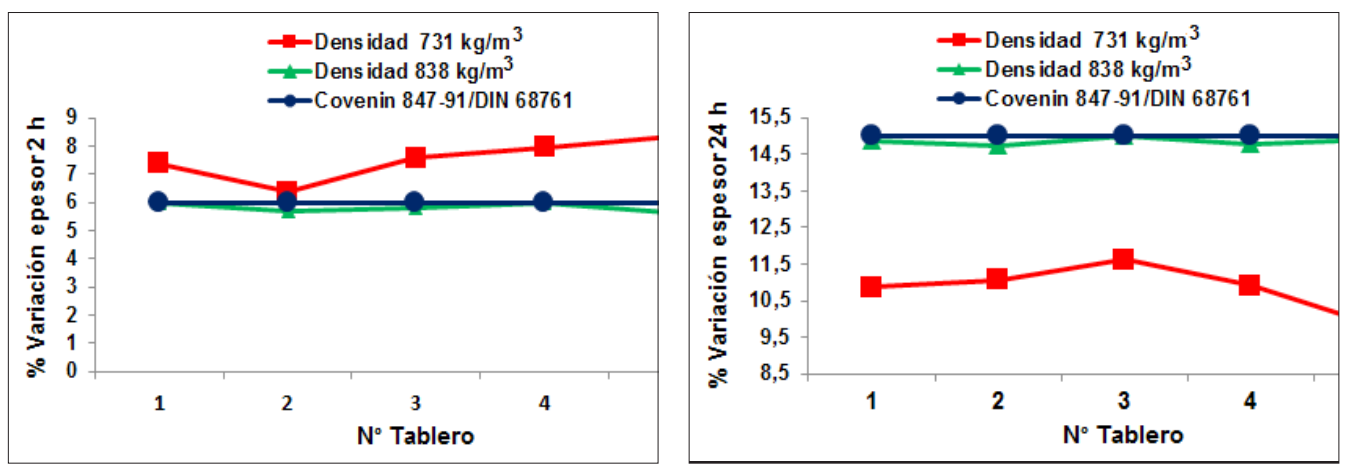

Figura 4. Variación de espesor o hinchamiento por inmersión en agua a) 2 h b) $24 \mathrm{~h}$, para tableros homogéneos de partículas de Eucalyptus urophylla. De acuerdo a las normas COVENIN (1991) y DIN (1981).

Los porcentajes de variación en espesor después de 24 horas de inmersión obtenidos para los tableros de E. urophylla son similares a los encontrados por De Almeida et al. (2015), quienes trabajaron con árboles de 10 años de edad de la misma especie, resina urea formaldehido al $8 \%$ de resinosidad, y obtuvieron tableros con una densidad real de $652 \mathrm{~kg} / \mathrm{m}^{3}$, encontrando porcentajes de variación de espesor del 18,7 \%. Un comportamiento similar fue encontrado por Naumann et al. (2008), quienes 
utilizaron madera de E. urophylla y fabricaron tableros de partículas con una densidad real promedio de $700 \mathrm{~kg} / \mathrm{m}^{3}$ cuyo porcentaje de variación del espesor fue de $16,8 \%$ para las 24 horas de inmersión.

Por otra parte, los resultados aquí obtenidos para la variación de espesor a 24 horas de inmersión están en concordancia con lo que exponen Durán (1981) y Moreno et al. $(2005,2010)$ que a mayor densidad del tablero, mayor será el hinchamiento; pero es contario a lo mencionado por Poblete y Peredo (1990) quienes no encontraron una correlación adecuada entre hinchamiento y densidad del tablero.

Aumentar el tiempo de inmersión a 24 horas, permite saturar paredes celulares que por su bajo contenido de humedad tienen capacidad de absorción produciendo un hinchamiento en las partículas, manifestándose finalmente en la variación del espesor del tablero. Como se ha mencionado anteriormente, existe variación de la densidad dentro del tablero, por lo que el hinchamiento del tablero tampoco será uniforme, es decir, el tablero se deforma y pierde estabilidad dimensional.

Gatchell et al. (1966), así como Roffael y Rauch (1972), manifiestan que incrementando la densidad del tablero podrían esperarse mayores hinchamientos. Sin embargo, de acuerdo con Lehmann (1960), el beneficio de obtener aumento de la resistencia mecánica es razón suficiente para compensar la tendencia de hinchamiento.

\section{Flexión estática (MOR)}

En la figura 5 se presentan los valores del MOR en flexión estática de los tableros de E. urophylla para ambas densidades. Los tableros con densidad de $731 \mathrm{~kg} / \mathrm{m}^{3}$ y $838 \mathrm{~kg} / \mathrm{m}^{3}$ superan en $57 \%$ y $78 \%$, respectivamente al valor mínimo de $17,65 \mathrm{MPa}\left(180 \mathrm{~kg} / \mathrm{cm}^{2}\right)$ establecido por las normas COVENIN (1991) y DIN (1981).

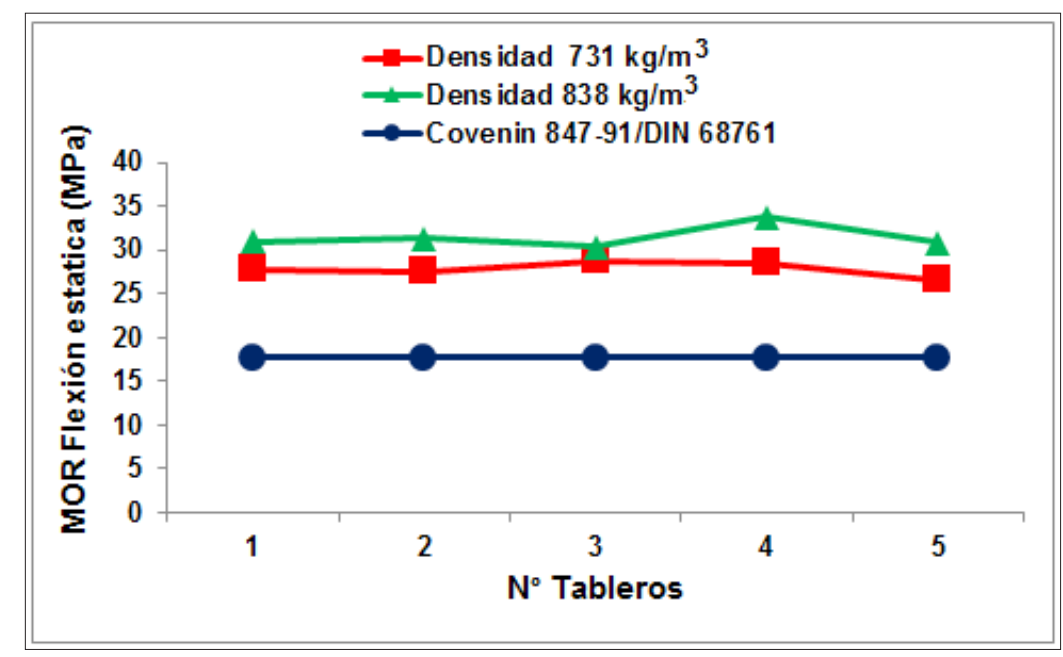

Figura 5. Módulo de ruptura (MOR) promedio en flexión estática para tableros homogéneos de partículas de Eucalyptus urophylla. De acuerdo a normas COVENIN (1991) y DIN (1981).

El módulo de ruptura (MOR) es aceptado como una medida de esfuerzo y representa la carga máxima de un espécimen que sometido a flexión llega a su fractura (Cai y Ross 2010). Sin embargo, la resistencia al esfuerzo en flexión debe ser considera hasta el límite elástico, para efectos de seguridad (McNatt 1973). Por otro lado, la adhesión interna o tensión perpendicular determina la resistencia del tablero a la delaminación o separación de sus capas (Cai y Ross 2010).

El MOR en flexión estática se incrementa a mayor densidad del tablero, esto se debe entre otros factores a una mayor eficiencia en la distribución de la resina y a un mejor contacto entre partículas-adhesivo, así como también a una mayor densificación de las partículas (mayor razón de 
compresibilidad), disminuyendo el volumen de espacios vacíos. Esto conlleva a que el tablero de mayor densidad trasmita los esfuerzos de partícula a partícula de manera más uniforme traduciéndolos en un aumento de la resistencia mecánica (Durán 1981, Maloney 1993, Bazyar et al. 2011).

En general, el MOR en flexión obtenido en este estudio con los tableros de ambas densidades (Figura 5), son mayores a los valores de tableros aglomerados de partículas encontrados de varias investigaciones, las cuales fueron realizadas en árboles y clones de la madera de E. urophylla, obteniendo tableros con densidades en el rango de $650 \mathrm{~kg} / \mathrm{m}^{3}$ a $800 \mathrm{~kg} / \mathrm{m}^{3}$ (Del Menezii et al. 1996, Mendonca 2008, Nauman 2008, Mendes 2009, Mendes 2014, De Almeida et al. 2015) y superando en un 55\% a los referidos por Iwari et al. 2000 y Bianche et al. 2012.

\section{Tensión perpendicular a la superficie del tablero}

En la figura 6 se presentan los valores obtenidos para la tensión perpendicular a la superficie de los tableros de E. urophylla para ambas densidades. Los tableros con densidad de $731 \mathrm{~kg} / \mathrm{m}^{3}$ y $838 \mathrm{~kg} / \mathrm{m}^{3}$ superan en $7 \%$ y $29 \%$ respectivamente, al valor mínimo de $0,35 \mathrm{MPa}\left(3,5 \mathrm{~kg} / \mathrm{cm}^{2}\right)$ solicitado por las normas COVENIN (1991) y DIN (1981).

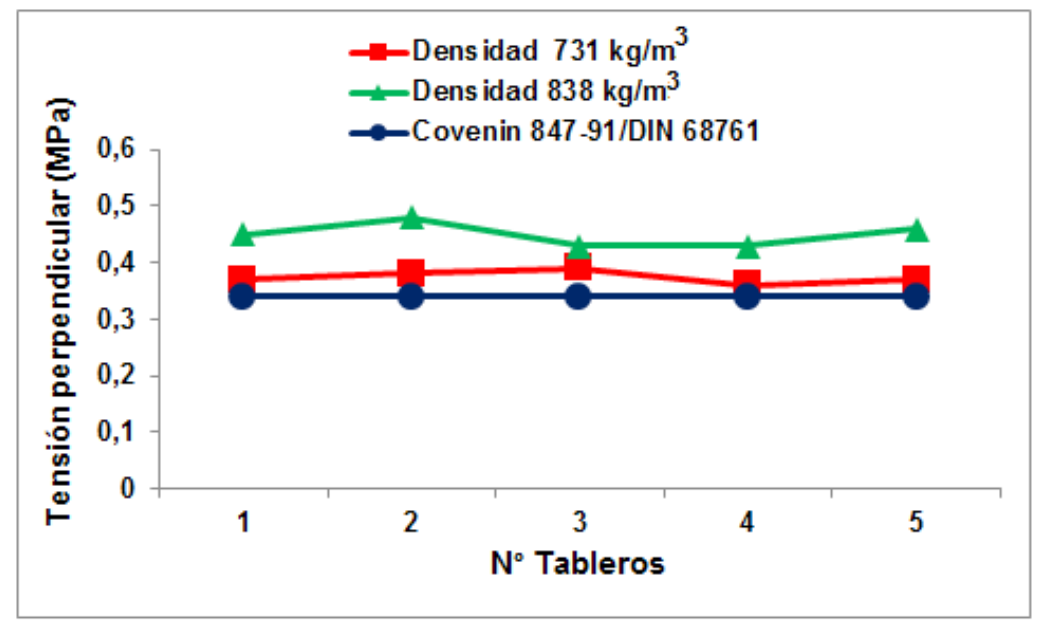

Figura 6. Tensión perpendicular promedio para tableros homogéneos de partículas de Eucalyptus urophylla. De acuerdo a normas COVENIN (1991) y DIN (1981).

En cuanto a la tensión perpendicular a la superficie del tablero (TPST), aumenta a medida que se incrementa la densidad del tablero y la relación de compresibilidad. Esto se puede atribuir al mayor contacto relativo entre las partículas y la mayor eficiencia de la resina, así como también a que se logra una densificación mayor en el centro del tablero debido al mayor volumen de partículas a ser prensadas. Por lo tanto, el gradiente de densidad en el espesor del tablero es menos variable que en el caso de los tableros de menor densidad (Durán 1982, Maloney 1993, Moreno et al. 2002).

En general, la adhesión interna obtenida en este estudio con los tableros de ambas densidades está dentro de los intervalos mencionados por Cai y Ross (2010) de $0,31 \mathrm{MPa}-1,17 \mathrm{MPa}\left(3,2 \mathrm{~kg} / \mathrm{cm}^{2}\right.$ a 12 $\mathrm{kg} / \mathrm{cm}^{2}$ ) para tableros de partículas de tipo industrial con densidad de $690 \mathrm{~kg} / \mathrm{m}^{3}$. De igual manera los resultados presentan similitud con los encontrados en varias investigaciones, utilizando madera de $E$. urophylla para fabricar tableros con densidades reales entre los $600 \mathrm{~kg} / \mathrm{m}^{3}$ y $750 \mathrm{~kg} / \mathrm{m}^{3}$ (Mendes et al. 2009, Bianche et al. 2012, De Almeida et al. 2015). 


\section{CONCLUSIONES}

Como se demostró en el análisis de varianza y en los resultados obtenidos, la densidad del tablero es un factor que influye de manera directa sobre las propiedades físico-mecánicas de un tablero aglomerado de partículas.

El aprovechamiento de la madera de la especie Eucalyptus urophylla, mostró buenos resultados y propiedades tecnológicas adecuadas para su trasformación en un producto de ingeniería como el tablero homogéneo de una capa de partículas con densidad media. Los tableros de este estudio cumplieron con los valores mínimos solicitados por las normas, en particular, para las propiedades mecánicas de MOR y adhesión interna. Sin embargo, los porcentajes de absorción de humedad y variación de espesor en algunos casos no cumplieron con los valores máximos aceptados por norma.

De acuerdo con los resultados obtenidos, el tablero homogéneo de partículas de E. urophylla con una densidad real de $838 \mathrm{~kg} / \mathrm{m}^{3}$ presentó las mejores características en cuanto a propiedades mecánicas de MOR, adhesión interna y mejor comportamiento en la absorción de agua y variación de espesor a 2 y 24 horas.

Finalmente, los tableros aglomerados de partículas de E. urophylla homogéneos de una capa, encolados con urea formaldehído y de ambas densidades poseen resistencia mecánica y adhesión interna aceptadas por las normas, por lo que se recomienda su uso en partes internas de muebles y gabinetes, como material decorativo superpuesto sobre otros materiales, como recubrimiento interno de casas prefabricadas, entre otros usos; siempre y cuando el tablero preste su servicio en sitios con poca o nula exposición directa a la humedad.

\section{BIBLIOGRAFÍA}

Ashoria, A.; Nourbakhsh, A. 2008. Effect of press cycle time and resin content on physical and mechanical properties of particleboard panels made from the underutilized low-quality raw materials. Industrial Crops and Products 28(2): 225-230.

Bazyar, B.; Tichi, A.; Rangavar, H. 2011. Particleboard made from fast growing Aspen wood and old rail road ties. Australian Journal of Basic and Applied Sciences 5(8): 548-553.

Bianche, J.; Carneiro, A.; Vital, B.; Pereira, F.; Dos Santos, R.; Soratto, D. 2012. Propriedades de painéis aglomerados fabricados com partículas de eucalipto (Eucalyptus urophylla), Paricá (Schizolobium amazonicum) e Vassoura (Sida spp.). Cerne 18(4): 623-630.

Cabral, P.; Vital, B.; Della, L.; Pimenta, A. 2007. Propriedades de chapas de aglomerado confeccionadas com misturas de partículas de Eucalyptus spp e Pinus elliottii ev. Árvore 31 (5): 897905.

Cai, Z.; Ross, R. 2010. Mechanical properties of wood-based composite materials. In: R. J. Ross, ed. Wood handbook-Wood as an engineering material. Centennial edition. General Technical Report FPL-GTR-190. Department of Agriculture, Forest Service, Forest Products Laboratory. Madison, Wisconsin, EUA. 12 p.

Comisión Venezolana de Normas Industriales. COVENIN. 1991. Norma venezolana para tableros de partículas de madera (provisional). COVENIN-847-91. Ministerio de Fomento. CaracasVenezuela.

De Almeida, K.; De Souza, K.; Mendes, R.; Junior, J.; Mendes, L. 2015. Qualidade de painéis aglomerados produzidos com Eucalyptus urophylla e resíduos da desrama de Acacia mangium Willd. Scientia Forestalis 43(107): 713-720. 
Del Menezzi, C.; De Souza, M.; Gonzales, C. 1996. Fabricacao e avaliacao tecnología da chapa aglomerada de mistura de Eucalyptus urophylla. TS. blake e Pinus oocarpa Schiede. Arvore 20(3): 371-379.

Deutsches Institut fur Normung. DIN. 1965a. Testing of wood chipboards; general requirements, sampling, evaluation. German National Standard. DIN 52360.

Deutsches Institut fur Normung. DIN. 1965b. Testing of wood chipboards; determination of dimensions, raw density and moisture content. German National Standard. DIN 52361. 2p.

Deutsches Institut fur Normung. DIN. 1965c. Testing of wood chipboards; bending test, determination of bending strength. German National Standard. DIN 52362. 2p.

Deutsches Institut fur Normung. DIN. 1965d. Testing of wood chipboards; determination of variation in thickness due to moisture. German National Standard. DIN 52364. 1p.

Deutsches Institut fur Normung. DIN. 1965e. Testing of wood chipboards; determination of tensile strength vertical to the chipboard plane. German National Standard. DIN 52365. 1p.

Deutsches Institut fur Normung. DIN. 1976. Testing of wood; determination of density. German National Standard. DIN 52182. 3p.

Deutsches Institut fur Normung. DIN. 1981. Particle boards; flat pressed boards for general purposes; FPO-board. German National Standard. DIN 68761.

Durán, J. 1981. Utilización de los aclareos de las especies de la plantación de Caparo para tableros aglomerados de partículas: Parte I, Teca. Universidad de Los Andes, Laboratorio Nacional de Productos Forestales. Mérida, Venezuela. 77 p.

Durán, J. 1982. Utilización de los aclareos de las especies de la plantación de Caparo para tableros aglomerados de partículas: Parte II, Gmelina. Universidad de Los Andes, Laboratorio Nacional de Productos Forestales. Mérida, Venezuela. 44 p.

Garay, D. 1988. Producción de tableros aglomerados de partículas a partir de mezclas de especies de los llanos occidentales. Centro de Estudios Forestales de Postgrado, Facultad de Ciencias Forestales y Ambientales, Universidad de Los Andes. Mérida, Venezuela.

Garay, D. 1997. Tableros aglomerados de partículas. Laboratorio Nacional de Productos Forestales, Facultad de Ciencias Forestales y Ambientales, Universidad de Los Andes. Mérida, Venezuela.

Gatchell, G.; Heebink, B.; Hefty, F. 1966. Influence of component variables on the properties of particleboard for exterior use. Forest Products Journal 16(4): 46-59.

Gonzales, L. 2016. Maderas del Orinoco, C.A. Proceso Producción Forestal. Ministerio del Poder popular para Industrias Básicas, estrategias sociales. Maturín-Venezuela.

Iwakiri, S.; Da Cruz, Clair.; Olandoski, D.; Brand, M. 2000. Utilização de resíduos de serraria na produção de chapas de madeira aglomerada de Eucalyptus saligna, Eucalyptus citriodora e Eucalyptus pilularis. Floresta e Ambiente 7(1): 251-256.

Kord, B.; Zare, H.; Abdollah, H. 2016. Evaluation of the mechanical and physical properties of particleboard manufactured from canola (Brassica napus) straws. Maderas- Cienc Tecnol 18(1): 9-18.

Lehmann, F. 1960. The effects of moisture content, board density and press temperature on the dimensional and strength properties of flat-pressed flakeboard. School Forestry, North Carolina State Univ., Raleigh, N.C. 106 pp.

Maloney, T. 1993. Modern particleboard and dry-process fiberboard manufacturing. Miller Freeman Inc., San Francisco, California, EUA. 686 p.

McNatt, J. 1973. Basic engineering properties of particleboard. Res. Pap. FPL-206. Madison, WI: U.S. Department of Agriculture, Forest Service, Forest Products Laboratory. 14 p. 
Medina, A. 1975. Seminario sobre proceso de fabricación de tableros de partículas de madera. Facultad de Ciencias Forestales y Ambientales, Universidad de Los Andes. Mérida-Venezuela.

Mendes, R.; Mendes, L.; Mendonça, L.; Júnior, J.; Mori, F. 2014. Qualidade de paines aglomerados homogêneos produzidos com a madeira de clones de Eucalyptus urophylla. Cerne 20(2): 329-336.

Mendes, L.; Iwakiri, S.; Mori, F.; Junior, J.; Mendes, R. 2009. Eucalyptus urophylla Stands wood utilization at two different ages for production of particleboard panels. Cerne 15(3): 288-294.

Mendonça, L. 2008. Produção de painéis de madeira com clones de Eucalyptus urophylla: estudo de caso para uso na indústria de móveis e construção civil. Dissertação de Mestrado. Engenharia de Materiais da Rede Temática em Engenharia de Materiais. Universidade Federal de Ouro Preto, Centro Tecnológico de Minas Gerais, Universidade do Estado de Minas Gerais. Belo Horizonte, Brasil.

Moreno, P.; Durán, J.; Garay, D. 2002. Efecto de las Sales CCA sobre las propiedades físicas y mecánicas en tableros de partículas de pino caribe. Revista Forestal Latinoamericana 17(1): 1-34.

Moreno, P.; Durán, J.; Garay, D.; Valero, S.; Shakespeare, T.; Nieto, R. 2005. Utilización de la madera de Fraxinus americana (fresno) en la fabricación de tableros de partículas. Revista Theoria 14(2): 57-64.

Moreno, P.; Durán, J.; Garay, D.; Valero, S.; Shakespeare, T. 2010. Utilización de tallos de Guadua angustifolia en la fabricación de tableros de partículas. Forest Systems 19(2): 241-248.

Moslemi, A. 1974. Particleboard. Vol. I Materials. Southern Illinois University Press. 239 p.

Naumann, R.; Vital, B.; Carneiro, A.; Lucia, R.; Silva, J.; Carvalho, A.; Colli, A. 2008. Propriedades de chapas fabricadas com partículas de madeira de Eucalyptus urophylla S. T. Blake e de Schizolobium amazonicum Herb. Árvore 32 (6): 1143-1150.

Peredo, M. 1988. Fabricación de tableros de partículas para uso exterior. Bosque 9(1): 35-42.

Peredo, M.; Torres, E. 1991. Fabricación de tableros de partículas con desechos industriales. Bosque 12(1): 49-57.

Poblete, H.; Peredo, M. 1990. Tableros de desechos del debobinado de especies chilenas. Bosque 11(2): 45-58.

Poblete, H.; Loncomilla, E.; Inzunza, L. 2000. Densidad del tablero de partículas y estabilidad dimensional. Bosque 21(2): 69-83.

Rocha, B.; Lehmann, W.; Sidney, R. 1974. How species and board densities affect properties of exotic hardwood particleboards. Forest Product Journal 24(12): 37-45.

Roffael, E.; Rauch, W. 1972. Influence of density on the swelling behavior of the phenolic resin bonded particleboards. European Journal of Wood and Wood Products 30(1): 178-181.

Stark, N.; Cai, Z.; Carll, C. 2010. Wood-based composite materials panel products, gluedlaminated timber, structural composite lumber, and wood-nonwood composite materials. In: R.J. Ross, ed. Wood handbook-Wood as an engineering material. Centennial edition. General Technical Report FPL-GTR-190. Department of Agriculture, Forest Service, Forest Products Laboratory. Madison, Wisconsin, EUA p:1-28.

Trianoski, R.; Iwakiri, S.; De Matos, J.; Prata, J. 2010. Avaliação de espécies alternativas de rápido crescimento para produção de painéis de madeira aglomerada de três camadas. Scientia Forestalis 39(89): 97-104.

Zambrano, L.; Moreno, P.; Muñoz, F.; Durán, J.; Garay, D.; Valero, S. 2013. Tableros de partículas fabricados con residuos industriales de madera de Pinus patula. Madera y Bosques 19(3): 65-80. 